\section{Toward a synthesis of genotypic typing and phenotypic inference in the genomics era}

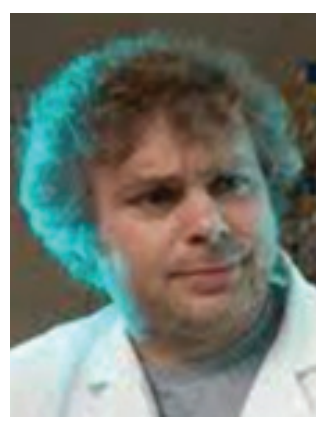

\author{
“...there is a need to safeguard against \\ squandering the power and novelty of WGS \\ data as a consequence of allowing our \\ thinking to be unnecessarily constrained by \\ the limitations of legacy typing techniques."
}

\section{Edward J Feil*}

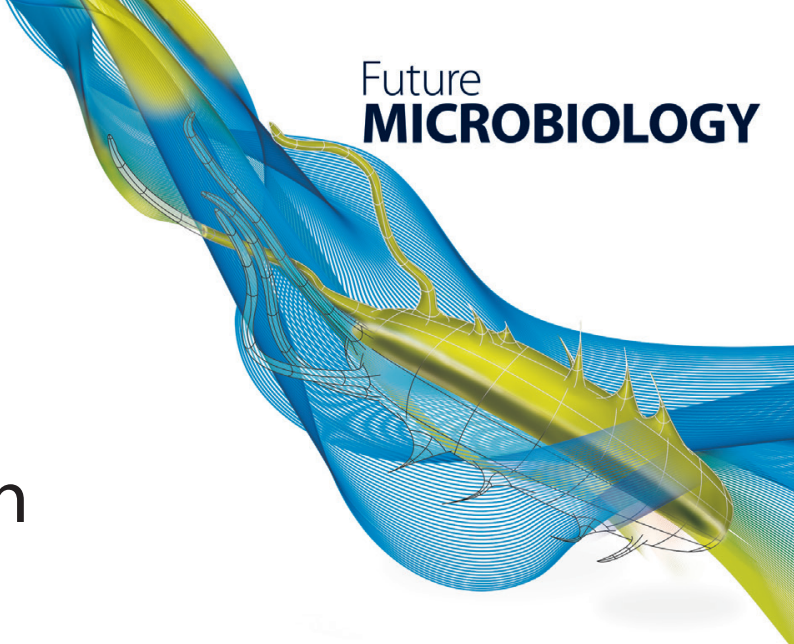

The development of techniques for assaying molecular variation within populations of bacterial pathogens dates back to the early 1970 s, when the first surveys based on protein electrophoretic mobilities in starch gels were carried out (multilocus enzyme electrophoresis [MLEE]) [1]. The subsequent decades witnessed a plethora of approaches, most notably Random Amplified Polymorphic DNA (RAPDs), Variable Number Tandem Repeats (VNTRs), Pulsed-Field Gel Electrophoresis (PFGE), Multilocus Sequence Typing (MLST), plus many species-specific variants of these methodologies. This technological diversification, memorably satirized by Mark Achtman [2], reflected in part the fact that all these methods, to greater or lesser degrees, represented compromises and trade-offs between resolution, repeatability, affordability and utility for population genetics. No single technique was universally applicable for all taxa, budgets and over all epidemiological scales. However, the advent of next-generation sequencing technology marks a fundamental shift away from the YATMs (Yet Another Typing Method), TATBSTMs (Tried and True But Stodgy
Typing Method) and TBCAs (Totally Boring Clonal Analysis) of yore [2], and paves the way for a much closer synthesis between genotype and phenotype.

Excluding the intriguing possibility of assaying patterns of DNA modification, whole-genome sequence (WGS) data approaches the 'ultimate' typing methodology, in the sense that there remain few further sources of variation left untapped. In recognition of this self-evident truth, there is a need to safeguard against squandering the power and novelty of WGS data as a consequence of allowing our thinking to be unnecessarily constrained by the limitations of legacy typing techniques. In fact, the added value of WGS data for inferring transmission over all epidemiological scales, from a single hospital ward to the entire planet, when combined with the opportunity to simultaneously infer phenotypes such as virulence and drug resistance, points to a near-future where the assignment of isolates to relatively broad-brush lineages in the absence of any meaningful phenotypic context, which is the purview of traditional typing, will seem hopelessly anachronistic.

\section{KEYWORDS}

- molecular epidemiology

- phenotypes • typing • wholegenome sequencing

*The Milner Centre for Evolution, Department of Biology \& Biochemistry, University of Bath, Claverton Down, Bath, BA2 7AY, UK; Tel.: +44 1225 383021; e.feil@bath.ac.uk

Future Medicine $\mathrm{fSg}$ 


\section{"Currently we have no conceptual framework, or even rules of thumb, by which to standardize the detection, demarcation or nomenclature of 'lowest- level types'; the sub-sub strains that represent fundamental units of epidemiology or adaptation."}

As a typing tool, one key advantage of WGS data over legacy techniques stands out: an unparalleled degree of discrimination. This has provided the ability to explore the spatial and temporal dynamics of sub-variants within single 'strains', previously defined as unique pulsotypes or multilocus sequence types (STs; [3]). Genomic and epidemiological dynamics can now be monitored or reconstructed using WGS data over weeks or months, within single hosts, or within individual hospitals or cities $[4,5]$. The geographical distribution of fine-scaled sequence clusters is set to form a key component of WGS-based epidemiology, and community-oriented tools such as microreact.org provide the means to explore the phylogenetic signals within a spatial framework. Thus, for most taxa, established clones, or multilocus STs can be subdivided by WGS data into increasingly finer phylogenetic and geographical scales, until the point is reached where isolates are found to be identical or which differ at, say, $<3$ SNPs across the genome. These finest-scale irreducible clusters likely represent a single outbreak, host or healthcare setting.

Although a key strength of WGS data, the ability to dig down to the finest phylogenetic scale, also poses a problem. Currently we have no conceptual framework, or even rules of thumb, by which to standardize the detection, demarcation or nomenclature of 'lowest-level types'; the sub-sub strains that represent fundamental units of epidemiology or adaptation. For most species, a pair of isolates differing at only a single SNP will typically not be considered as effectively different, particularly if the SNP in question does not have any obvious functional consequences. However, this may simply reflect our ignorance of the underlying genetics. If, for example, a single intergenic SNP happened to alter the expression of a global regulator with commensurate knock-on effects for virulence, then it may well be justified to consider this particular variant as distinct, perhaps even deserving of a name. On the other hand, it clearly makes no sense to categorize everything as being different from everything else on the off-chance that the microvariation we observe might be meaningful, particularly as we will encounter the vast majority of these putative microtypes only once. Unlocking the full utility of WGS data at this fine-scale therefore requires that phenotypic context can no longer been seen as an optional embellishment of typing data. Rather, at this quantum level of bacterial evolution the meaningful demarcation of WGS 'types' becomes dependent on our ability to understand the functional relevance of the microvariation we observe.

Critics may raise two objections at this point: How realistic is it to infer clinically or epidemiologically relevant phenotypes from WGS data?, and What actually is the problem with deploying WGS data simply as a high-powered typing tool anyway? I shall address these in turn. First, many different types of genomic variation can have implications for phenotype. Above I discussed SNPs within the core genome, but changes in gene content are probably even more pertinent due to the fact that many clinically relevant genes (such as toxins, adhesins, resistance cassettes) are carried on mobile genetic elements (MGEs) [6]. The gain and loss of such genes probably represent the most robust and tractable means to predict clinical traits from genome data, and continuing improvement in de novo assembly software means that assaying the presence of both known and novel genes is becoming more routine [7]. Recent studies have demonstrated the use of genome-wide association (GWAS) approaches to link specific genes or elements with particular phenotypes [8], and it may be that monitoring the epidemiology and spread of highly mobile MGEs such as plasmids and phage becomes just as pertinent for the protection of public health as tracking the movement of the host bacteria. Core genome SNPs present a more complex case, but again a recent GWAS study has proved useful in linking individual SNPs with virulence in MRSA (notably, a number of the implicated SNPs fell within intergenic regions) [9].

In the case of Staphylococcus aureus, antibiotic resistance profiles can be accurately predicted from WGS data [10], and a number of tools are being developed for this purpose [11]. Given a representative sample, evidence concerning the risk to public health of newly emergent lineages can also be discerned at a population level by examining the spatial and temporal distribution of specific clusters. The topology of the phylogenetic trees can also yield fruitful evidence; short internal nodes and long terminal branches (i.e., a star-like phylogeny) being indicative of rapid population expansion and spread. Other types of genomic variation, such as genome rearrangements and small INDELS are also likely to be phenotypically relevant though are currently more difficult to reconstruct from short read NGS data. However, de novo assembly and 
mapping software is improving constantly, and alternative platforms are becoming available that generate much longer reads [12].

As for the second question, there is nothing wrong with using WGS data simply as a highpowered typing tool, except that it lacks ambition. The point is not only that WGS-defined clusters can occasionally be supplemented with inferred phenotypes, but more that these two perspectives are most productively considered as a synthesized whole (after all they should derive from exactly the same source data). The development of WGS databases on a 'gene-bygene' model [13], which essentially treats genome data as a massively extended MLST scheme, is a convenient and scalable approach for storing and interrogating WGS data for typing purposes [14]. However, such an approach is not optimal for establishing genotype phenotype links, as key sources of variation of potential phenotypic relevance, such as intergenic sites, are, by default, excluded, as is all positional information. Using WGS as if it were a conventional typing tool it may be possible to infer, say, that a particular variant of MRSA was transmitted from hospital $\mathrm{X}$ to hospital Y. But how much better if we could also simultaneously deduce, without recourse to a separate analysis, that this variant also had low level of resistance to disinfectants, or produced a specific toxin under certain conditions, or was prone to colonize a particular body site.
The synthesis of genotypic typing and phenotypic inference based on WGS data, as outlined here, is far from straightforward. The initial establishment of genotype phenotype links often requires complex statistical analysis [15] and rich experimental data and/or clinical and epidemiological metadata. There are commensurate technical challenges in the design and implementation of databases and analytical tools that can account for core SNPs, gene content changes and other types of genomic variation, and ultimately the generation of the actual genome sequence becomes a relatively trivial piece of the jigsaw. Nevertheless, the successes so far, in particular the remarkable accuracy by which resistance phenotypes in $S$. aureus can be predicted from sequence data alone, should encourage us to begin thinking along these terms, and to move epidemiological typing beyond a rather conceptually limited exercise in classification.

\section{Financial \& competing interests disclosure \\ The author has no relevant affliations or financial involve- ment with any organization or entity with a financial inter- est in or financial conflict with the subject matter or materi- als discussed in the manuscript. This includes employment, consultancies, honoraria, stock ownership or options, expert testimony, grants or patents received or pending, or royalties. \\ No writing assistance was utilized in the production of this manuscript.}

\section{References}

1 Milkman R. Electrophoretic variation in Escherichia coli from natural sources. Science 182(4116), 1024-1026 (1973).

2 Achtman M. A surfeit of YATMs? J. Clin. Microbiol. 34(7), 1870 (1996).

3 Maiden M, Bygraves J, Feil E et al. Multilocus sequence typing: a portable approach to the identification of clones within populations of pathogenic microorganisms. Proc. Natl Acad. Sci. USA 95(6), 3140-3145 (1998).

4 Harris S, Feil E, Holden M et al. Evolution of MRSA during hospital transmission and intercontinental spread. Science 327(5964), 469-474 (2010).

5 Golubchik T, Batty E, Miller R et al. Within-host evolution of Staphylococcus aureus during asymptomatic carriage. PLoS ONE 8(5), e61319 (2013).
6 Penadés JR, Chen J, Quiles-Puchalt N, Carpena N, Novick R. Bacteriophagemediated spread of bacterial virulence genes. Curr. Opin. Microbiol. 23, 171-178 (2015).

7 Kisand V, Lettieri T. Genome sequencing of bacteria: sequencing, de novo assembly and rapid analysis using open source tools. BMC Genomics 14(1), 211 (2013).

8 Sheppard S, Didelot X, Meric G et al. Genome-wide association study identifies vitamin B5 biosynthesis as a host specificity factor in Campylobacter. Proc. Natl Acad. Sci. USA 110(29), 11923-11927 (2013).

9 Laabei M, Recker M, Rudkin J et al. Predicting the virulence of MRSA from its genome sequence. Genome Res. 24(5), 839-849 (2014).

10 Gordon N, Price J, Cole K et al. Prediction of Staphylococcus aureus antimicrobial resistance by whole-genome sequencing. J. Clin. Microbiol. 52(4), 1182-1191 (2014).
11 Bradley P, Gordon N, Walker T et al. Rapid antibiotic resistance predictions from genome sequence data for $S$. aureus and $M$. tuberculosis. doi:http://dx.doi.org/10.1101/018564 (2015) (Epub ahead of print).

12 Madoui M, Engelen S, Cruaud C et al. Genome assembly using Nanopore-guided long and error-free DNA reads. BMC Genomics 16(1) (2015).

13 Maiden M, van Rensburg M, Bray J et al. MLST revisited: the gene-by-gene approach to bacterial genomics. Nat. Rev. Microbiol. 11(10), 728-736 (2013).

14 Jolley K, Maiden M. Using MLST to study bacterial variation: prospects in the genomic era. Future Microbiol. 9(5), 623-630 (2014).

15 Marttinen P, Gillberg J, Havulinna A, Corander J, Kaski S. Genome-wide association studies with high-dimensional phenotypes. Stat. Appl. Genet. Mol. Biol. 12(4) (2013). 